\title{
Global Solutions to the Challenges of Setting up and Managing a Stem Cell Laboratory
}

\author{
Maneesha S. Inamdar • Lyn Healy • Abhishek Sinha • \\ Glyn Stacey
}

Published online: 29 October 2011

(C) The Author(s) 2011. This article is published with open access at Springerlink.com

Keywords Stem cell laboratory establishment - Laboratory design - Laboratory operation - Good cell culture practice . Laboratory management $\cdot$ Standardization $\cdot$ Quality control

\section{Background and Origin}

In recent years, stem cell research has made major contributions to our understanding of biology. The ability to reprogramme somatic cells to stem cells of desired potency has made this exciting area of research accessible to almost every laboratory, in spite of varying ethical, political and economic scenarios. The promise of stem cell research in making regenerative medicine accessible has further attracted clinicians, materials scientists, chemists, physicists and other non-specialists to this field of research. While this situation is desirable, the novice usually faces the daunting task of setting up and maintaining a stem cell laboratory, often without access to local expertise. Researchers allocate a significant amount of resource to keep their approach technologically advanced. Delivery of robust and reliable data (i.e. achieving reliable and reproducible stem cell cultures for experimentation) is often neglected. This can result in disruption and delay in laboratory work and at worst, wasted research resource and even retraction of publications. This document lays out fundamental

\footnotetext{
M. S. Inamdar $(\bowtie) \cdot$ A. Sinha

Jawaharlal Nehru Centre for Advanced Scientific Research, Jakkur P.O,

Bangalore 560064, India

e-mail: inamdar@jncasr.ac.in

L. Healy $\cdot$ G. Stacey

UK Stem Cell Bank, National Institute for Biological Standards and Control (NIBSC-HPA),

Blanche Lane, South Mimms,

Herts, UK

issues to be addressed in the establishment of a stem cell culture laboratory. The aim is to provide guidance on ways to overcome many challenges to smooth operation, encountered in varying climates and environments. Parts of this overview are modeled on the Guidance on Good Cell Culture Practice [3] and should be considered as an 'aide memoire' to complement existing guidelines. This guidance originated from experience gained by the authors in the establishment of multiple cell culture laboratories and training students in different countries with widely different environmental conditions in Northern Europe and in India.

\section{Scope}

This document addresses the full range of issues that new as well as established stem cell researchers charged with setting up a stem cell laboratory may face. It proposes solutions to deal with potential problems ahead of time. The aim is to help increase reproducibility of procedures, reduce uncertainties in supply, and help academics meet international scientific and ethical requirements.

\section{Introduction}

Good scientific practice and maintenance of high standards of mammalian cell culture is important for any research based on the use of stem cell lines. Contributions to stem cell research are now global and include researchers and countries that are relatively new to the field. One important goal is to establish consistent standards of scientific and technical competence in stem cell culture that will promote good science and efficient use of research resources. This paper identifies generic guidance for establishment and 
management of stem cell culture laboratories. It also recognizes the challenges that exist for laboratory workers in countries with a developing infrastructure where constraints on local supply and maintenance support can divert time and effort away from core research activity. Relocating a laboratory to a new environment poses challenges that a researcher has to prepare for. This paper provides an outline of the range of issues, one should anticipate in attempting to establish a new stem cell culture facility equipped and staffed to deliver the robust data required for frontier research.

Most principal investigators trained in established, sophisticated facilities find themselves in a situation where they make all efforts to establish equally good laboratories but cannot duplicate their experimental procedures successfully. Investigation of such problems often leads to the conclusion that "bad air and water" are to blame. While these are important, especially in a tropical or otherwise humid climate, usually a combination of several parameters needs to be resolved. Awareness of these factors and persistence in tackling them systematically is essential for smooth operation of the laboratory. This guidance equips the researcher with prior knowledge and strategies to avoid or overcome undesirable situations.

Two scenarios are particularly relevant for stem cell research: 1] Primary cultures are often derived from tissues where one has no- or limited control over how the tissue was obtained and the potential infection risk it carries 2] Cultures are often carried continuously for long periods, several years in some instances. These conditions necessitate meticulous planning and reproducible execution of culture protocols and careful aseptic technique to maintain the health and integrity of the cells and safety of laboratory workers. The measures suggested here are much the same for all routine cell culture and should be implemented before embarking on stem cell culture.

\section{Some Common Myths of Stem Cell Culture Practice}

An informal survey of several stem cell laboratories, training of over 80 researchers and review of several grant applications has helped generate this list of common misconceptions regarding best practice in stem cell culture:

Statement 1. "Good stem cell culture can be practiced only in a room with sterile filtered air."

Statement 2. "A room with filtered air supply is sterile during routine use and hence one is unlikely to get contamination."

Response to Statements $1 \& 2$ Cell culture material can be maintained aseptically and free of contamination without the need for completely sterile laboratory facilities. A range of techniques and skills can be employed in combination to sustain uncontaminated cell cultures; these include optimized and robust techniques with accurate standard operating procedures (SOPs). Whilst contamination may arise from the general laboratory environment, one of the most concentrated sources of microbial contamination is the human body. Key elements in the maintenance of contamination- free cultures include the correct use of laboratory clothing, effective sterilization of cell culture equipment and media, effective disinfection policies and proficiency of staff in good aseptic technique.

Statement 3. "A cGMP facility is essential for all stem cell research."

Response to Statement 3 cGMP (current Good Manufacturing Practices) is not required for stem cell research. 'cGMP' is a way of working that is specific to the formulation of a product [4]. GMP is neither necessary nor designed to accommodate research procedures. Some quality systems such as ISO9000 are set up to accommodate R\&D under the scope of product design. However, before embarking on implementation of such systems the need and cost involved should be carefully considered. Typically, GMP refers to the procedures adopted to show that a particular set of protocols meets specified standards and ensures that delivery of a defined final product is successful, well documented and validated. GMP is used to encompass the clean facilities required for cell culture and in particular, for establishing cells that might be used for manufacture of medicinal products or human therapy. If cells are being isolated and processed for use in humans, it is wise to have the process of cell procurement (obtaining consent, tissue harvesting), preservation and storage carried out under well- documented conditions. Documents should contain evidence that the material is maintained such that growth of endogenous contamination or introduction of contamination during processing is highly unlikely. Specific standards for environmental and process control requirements for banking and processing human tissues have been established and would be a good example to follow for production of cells and tissues for human application (e.g. EUTCD, US CFR 21 part 1270 \& 1271) (http://www.accessdata.fda.gov/scripts/cdrh/ cfdocs/cfcfr/CFRSearch.cfm?CFRPart=1270/1271).

Statement 4. "The major problem in having a cGMP facility is the high cost of setting it up."

Response to Statement 4 It is true that the cost of establishing a GMP facility is high. However, following establishment of a new facility, the ongoing cost of keeping such facilities running is very high as this includes cost of special services (validation), equipment, clean-room consumables and the large staff-time commitment to quality assurance. 
Statement 5. "Establishment of SOPs and records of experimental detail are required only for "important" experiments or for a cGMP laboratory."

Response to Statement 5 Accurate records of experimental work are critical to demonstrate how results were obtained and enable replication of results. A personal 'laboratory note book' in which all scientific experiments and results are recorded is generally used. Such records are also important to provide evidence for key discoveries and may be critical to filing successful patents. Key laboratory procedures change little over time and are the same for a range of projects e.g. preparation of certain buffers and media, operation of laboratory equipment. In such cases, formally documented SOPs are useful in any laboratory to ensure that:

- there are accurate descriptions available that document core technical procedures,

- laboratory staff have protocols that enable them to carry out procedures reproducibly thus enhancing reliability and comparability of data generated over time,

- it is possible to review data generated before and after changes were made in a particular procedure and thus review their impact on data.

All of these indications identify issues key to good scientific practice and are not solely required for commercial or highly regulated work. Any research laboratory should have a set of core protocols that document the key technical procedures. It is not unusual for protocols to develop with time and it is important to document in laboratory note books which version of a protocol is used and if there have been deviations from standard procedure. However, it is also important to recognise that the availability of detailed protocols is no substitute for staff training and competency.

\section{Points to Consider When Initiating and Setting up a Stem Cell Laboratory}

Researchers usually find themselves plunged into the construction or redesign of facilities with relatively little or no guidance or training. Given the fast pace of stem cell research there is an understandable urgency in starting up such a laboratory. However, a careful review of available infrastructure, facilities and resources would identify the level of support available for the new facility. This helps the researcher to assess and allow for any gaps or challenges in local laboratory support functions, and introduce measures to help ensure successful and timely delivery. In addition, visits to established laboratories doing similar work can provide valuable insights from user experiences. Approaches described here will aid researchers in laboratory design and help establish SOPs suited to their particular circumstances. Critical aspects of laboratory establishment and operational culture may be beyond the investigator's control. A formal framework to manage the delivery of a new facility will enable the researcher to recognize and plan for these issues as outlined in this guidance. Detailed advice for efficient completion of larger projects is also available for an example see the PRojects IN Controlled Environments (Prince 2 website at http://www.prince2.com/)

\section{Scope of Work and Facility Remit}

The first question that has to be asked is; "what is the laboratory expected to deliver?" A mismatch between the sponsor's expectations and what the principle investigator can deliver will inevitably lead to frustration and possibly failure to secure ongoing support from the sponsor. It is imperative that the investigator formally agrees to the expected overall outcomes with the Institution and/or sponsor. This could be in the form of an approved grant proposal, contract or tender document or some other formal agreement. Whatever form this takes, it should be sufficiently detailed to enable both sponsor and principle investigator to understand and agree upon the precise expected outcomes and timelines for a new facility. In particular this document should clearly describe what the laboratory must deliver in terms of anticipated output, types of work to be included and the standards to which the laboratory should operate. This process is an essential precursor to finalizing laboratory design and cost, which may vary from the original proposal and clearly must be discussed with the sponsor.

Furthermore, many changes may need to be implemented during financing, design and construction of a laboratory facility. It will be helpful to engage the sponsor periodically during the process to give confidence that the final facility will meet the researcher's needs and the sponsor's expectations. This is important as it is quite common for the needs of both the sponsor and responsible researcher to change over the period from planning to completion. This first evaluation should be developed into a specification for the facility (usually called a "user requirements specification" or URS) which identifies all elements of the desired facility, how they should relate to each other and any recognised formal standards (e.g. ISO14644, GLP, GMP) [5], laws and regulations that must be met. These should be discussed with any contractors to ensure that there is a clear understanding on what is required and how it will be achieved physically. Table 1 gives a brief description of the common quality standards that may be applied to cell culture work. 
Table 1 Some standards that can be applied to cell culture processes

\begin{tabular}{llc}
\hline Standard & General description & Application in cell culture \\
\hline ISO $9000^{2}$ & $\begin{array}{c}\text { General structure and operation } \\
\text { of a quality management system } \\
\text { Laboratory measurement and trends }\end{array}$ & $\begin{array}{c}\text { Any production process that uses cell culture and also research } \\
\text { work under the "design" element of the standard } \\
\text { ISO17025 }\end{array}$ \\
GLP (OECD) & Safety testing & $\begin{array}{c}\text { batch release } \\
\text { Testing cell cultures for viral contamination, genetic stability, } \\
\text { identity etc. }\end{array}$ \\
Good manufacturing practices & Control of the manufacturing process & Cell banking and scale-up procedures \\
\hline
\end{tabular}

A successful facility requires ongoing institutional support and hence running costs, which can be substantial for clean room laboratories, should be projected realistically and discussed with the sponsor at an early stage. While the short- term remit of the facility may be basic research, the long term expectations may well be the delivery of clinical grade materials for use in humans. There should be clarity on whether these materials would be produced within the proposed laboratory or as a separate programme/facility. In the former case at least some component of the facility must meet national requirements to deliver clinical grade material and the sponsor will need to be prepared for the cost of such facilities [1]. Equally, instigating the construction of a high quality and expensive clean room facility will be impressive but if the real requirement is for research publications, it is probably wise to focus resource on staff and equipment for that purpose.

\section{Location}

Often an investigator faces the task of redesigning existing space that was used for other activities. Matching the existing space and services with the new needs for laboratory layout and equipment should be considered carefully. In addition, previous laboratory activity (e.g. toxins, infectious agents) may have implications for the preparation and cleaning of the area prior to refurbishment. Hence, when fitting a new stem cell culture facility into existing space it is especially important to be aware of prior use and the precise requirements for the new stem cell culture laboratory.

Cell culture activity can be affected adversely by many factors. In general, the primary requirement for a cell culture laboratory is a clean and well- controlled environment. Any sources of heavy contamination such as dust, aerosols and damp areas will be problematic for cell culture e.g. waste disposal, drainage systems, large machinery, large- scale cooling systems. Other laboratory work involving culture of microorganisms or animal housing will also represent a contamination risk. Damp or low lying areas can aggravate the problems due to surface rain-water or a high water table. Where these are unavoidable appropriate controls in design and laboratory procedures should be applied. Even a modest laboratory size, should still allow for segregation of certain activities as outlined in Laboratory Design section below. Where the laboratory will need to be GMP compliant there will be further considerations in terms of available services and facilities and constraints on location particularly in terms of local sources of contamination.

Examples of potentially problematic laboratory features that may arise in retrofitting a cell culture facility largely relate to the requirements of equipment to provide clean air for aseptic processing and include:

- Direct access from a main corridor or to other unrelated areas of activity (this excludes fire exits which must take precedence in emergencies).

- Laboratories with significant dead-space areas (i.e. with no air flow provided by ventilation) which will inevitably collect air borne debris.

- Laboratories with low ceilings that may not provide sufficient space for replacement of HEPA filters in class II cabinets or could adversely affect the airflow to class II cabinets. These factors may hinder efficient exclusion of contamination during aseptic work.

- Very small or narrow labs which can affect correct class II cabinet function as well as hinder user movement, causing accidents.

- Laboratories with waste pipes/drainage/ventilation from areas handling high risk or contaminated material.

- Building material is also important as any sources of damp (such as porous walls or a leaking roof, rising damp) can cause serious and long term contamination problems

- Heavy equipment such as centrifuges and incubators should be located to permit thorough cleaning of lab walls, ceilings and floors and may be mounted on wheeled benches to facilitate cleaning.

An example of a cell culture laboratory layout showing segregation of different laboratory functions is given in Fig. 1. 


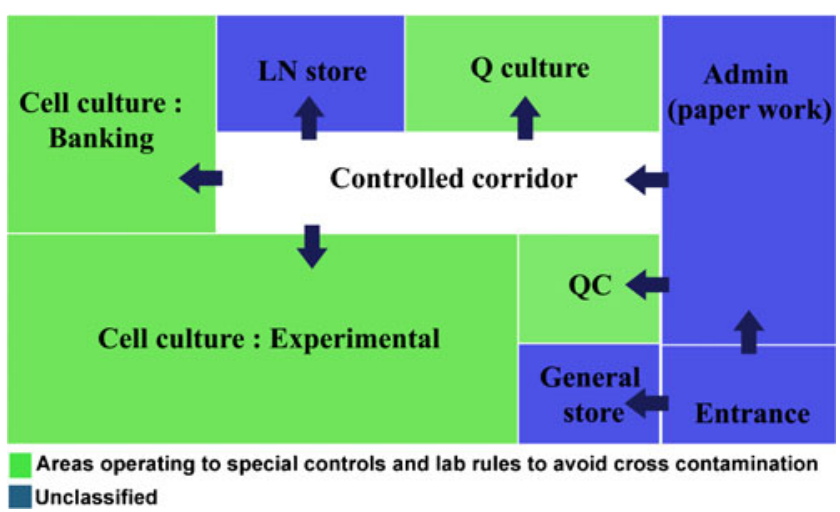

Fig. 1 Suggested layout for a stem cell culture laboratory. $\mathrm{LN}=$ Liquid Nitrogen; $\mathrm{Q}=$ Quarantine; $\mathrm{QC}=$ Quality Control

\section{Laboratory Design}

\section{Specification}

Once a contractor has been engaged to build the laboratory, the intended principal users of the new laboratory (e.g. the principal investigator, senior members of laboratory staff) should establish a written design specification that identifies the necessary engineering work for each area and how the laboratory areas will be coordinated. Laboratory layout, laboratory finishes, air quality etc. should be specified based on the intended purpose. This is important as adopting a strategy to set the highest standard for all areas, whilst attractive, may lead to waste of funds on areas in which it is difficult to do research and which could also incur unsustainable running costs. Criteria to be used for establishing an acceptable laboratory standard should be listed and may include nature of construction materials, air-borne particles, lighting, humidity range, integrity of laboratory boundaries with other areas and controlled access. SOPs should be established for acceptable movement of materials, staff and waste to minimise opportunities for contamination of clean products and new materials coming into the laboratory. Attention to ergonomics is also important as awkward or congested laboratory spaces are likely to suffer higher levels of accidents and contamination of aseptic cell culture work.

Important principles for key cell culture laboratory areas include:

- Cell culture areas should not be thoroughfares for laboratory "traffic" to minimise opportunities for cell culture contamination and disturbance of air flow.

- Areas where stocks or banks of cells are prepared for future use should be maintained at the highest standard of cleanliness reasonably possible given local conditions. These areas should be at the furthest point from the main entrance and waste storage or other activities carrying high risk of contamination.
- The cell culture laboratory should be a restricted area with controlled access to prevent practices that could increase risk of contamination and access by untrained staff. This can be facilitated by a 'clean' corridor controlling access between non-cell culture or office areas and all clean culture areas.

- Processing and incubation of microbiology QC samples should be carried out in an isolated area where any control or isolated organisms cannot contaminate aseptic cell culture work.

These principles are illustrated in the laboratory outline given in Fig. 1.

\section{Air Quality}

Clean rooms can be set up where space and funds permit. They provide significant advantages for the performance of cell culture work and will be vital where cells intended for human therapy require processing and culture. A 'clean room' is a laboratory designed to operate to a certain standard (usually ISO14644) for air quality with careful attention paid to the laboratory furniture and finishes that do not harbour or permit growth of microorganisms. Materials used should not release particles into the laboratory air and should withstand regular cleaning and disinfection. A typical design incorporates the 'onion skin' principal for air pressures/layout whereby laboratory pressures are managed to provide highest positive pressure in areas where processing gives greatest risk of contamination to cell cultures (for an example see [7]). Air quality is graded for different kinds of activity and a comparison of the major air quality standards is given in Table 2. For specialist manufacturing facility design the reader is referred to [15]. As already discussed above, the design will also depend on whether the scope of work is basic research only or research that might give rise to materials (e.g. cell lines) for future use in human therapy or production activities designed to supply preparations for routine clinical use.

For research involving stem cell cultures that will not be used in therapy, clean rooms of class 10,000 and below are not a requirement. The key issue in a research cell culture laboratory is to demonstrate that the laboratory staff can maintain cell culture for extended periods and in general without the use of antibiotics. Thus, key stem cell culture activity need not meet an international clean room standard but would require a combination of facilities and procedures which maintain a clean laboratory area that can be defined based on a local standard. Periodic checks with simple environmental screening methods such as 'settle' plates and surface swabs are sufficient to demonstrate that the laboratory maintains required standards. Some degree of contamination is unavoidable and "normal" limits of environmental contam- 
Table 2 Classifications used for clean areas for aseptic manufacturing processes

\begin{tabular}{|c|c|c|c|c|c|}
\hline EU grade & USA & SI & Description & $\begin{array}{l}\text { 'At rest' value for } \\
\text { maximum number } \\
\text { of particles } / \mathrm{m}^{3} \text { greater } \\
\text { than } 0.5 \mu \mathrm{m}\end{array}$ & $\begin{array}{l}\text { 'In operation' value } \\
\text { for maximum number } \\
\text { of particles } / \mathrm{m}^{3} \text { greater } \\
\text { than } 0.5 \mu \mathrm{m}\end{array}$ \\
\hline A & 100 & M3.5 & Open aseptic processes such as culture passage & 3,500 & 3,500 \\
\hline B & 100 & M3.5 & Background for grade A processes & 3,500 & 3,500 \\
\hline $\mathrm{C}$ & 10,000 & M5.5 & $\begin{array}{l}\text { Clean areas such as clean corridor providing } \\
\text { access to grade B zones }\end{array}$ & 350,000 & $3,500,000$ \\
\hline $\mathrm{D}$ & 100,000 & M6.5 & & $3,500,000$ & Not defined \\
\hline Unclassified & Unclassified & Unclassified & $\begin{array}{l}\text { Restricted access liquid nitrogen storage } \\
\text { and general access areas }\end{array}$ & - & \\
\hline
\end{tabular}

ination (e.g. number of colonies per settle plate) can be set locally that do not prevent clean antibiotic-free cell culture in class II cabinets.

Where materials developed or processed in the laboratory are intended for clinical use the ability to provide a Class 100 air quality for open processes (such as culture passage) is usually required. This is often achieved by performing all cell culture work in a Class 100 biological safety cabinet located in a clean room environment typically operating at Class 10,000. However, this air quality standard can also be achieved using an 'isolator' cabinet where the operators manipulate cultures through glove ports. Such rooms require a high level of monitoring and maintenance and a significant level of quality assurance, beyond strict implementation of SOPs; including restrictions on the type of clothing, personnel movement etc. to maintain the clean room environment. Any relaxation of procedural controls and SOPs would invalidate the clean room environment and any materials produced in it.

\section{Operational Considerations}

Contingency plans to tackle failure of various equipment (e.g. liquid nitrogen storage (see below), freezers, class II cabinets) should be established and documented.

It is important to consider sources of contamination such as water present in sinks, drains, waterbaths or air conditioners or high humidity equipment such as cell culture incubators. Air conditioners need regular maintenance and servicing, and should be located so as not to disturb laminar flow in biosafety cabinets while also providing easy access for service personnel. Incubators should be carefully maintained to prevent build up of contamination. If water trays are used to maintain humidity these should be regularly cleaned and treated to prevent contamination (e.g. copper sulphate crystals) and gas inlets should have in line filters which are replaced periodically. Incubators can also be purchased that have automatic heat disinfection cycles.

Water supply, sinks and water baths in the laboratory are best avoided as these environments are a rich source of microbes. Bacteria, fungi and microbial vectors (e.g. cockroaches, ants, centipedes and spiders) (e.g. [2, 6, 9]) may also multiply in damp cold storage rooms and large fridges which will require regular cleaning regimes to prevent the buildup of detritus and microbial contamination. Sinks and drains will harbour a range of environmental microorganisms at high concentrations that can also be resistant to antibiotics. They can also be a potential source of unfiltered air entering the laboratory under exceptional weather conditions causing back pressure in waste-pipes or plumbing failures. Even where water ingress and dampness is only temporary it may encourage growth of spore forming organisms. Spores can persist in locations in the fabric of the building which cannot be disinfected and may cause intermittent but persistent contamination problems.

Where water supply is deemed essential an area adjacent to the laboratory can be designated for a water inlet and sink. Alternatively disinfectant gels can be used for waterfree hand cleaning.

Liquid nitrogen storage areas have special requirements [10] and should be well-ventilated but also able to rapidly dissipate any gas released from normal use or significant liquid nitrogen spillages. Any environment that is poorly ventilated should not be used for liquid nitrogen storage. It is also advisable to fit exhaust-fans activated by low oxygen sensors. Storage areas should also be clean as contamination, including human pathogens, readily builds up in the storage vessels and can become a source of contamination when cells are recovered [17]. Freezers $\left(-80^{\circ} \mathrm{C}\right.$ etc. $)$ should not be kept in liquid nitrogen storage areas but in separate well ventilated areas.

\section{Services}

\section{Liquefied Gases}

A cell culture laboratory needs an assured supply of liquid nitrogen and $\mathrm{CO}_{2}$. Storage area requirements regarding 
contamination and safety issues and offsite nitrogen storage should be given careful attention (see above). Consideration should be given at the design stage to the provision for piped gas from outside the clean room area so that gas cylinders can be replaced without risk of introducing contamination to cell culture work. For gases it is advisable to allow for backup cylinders to enable manual or automatic switch over when each cylinder is exhausted. The gas should be piped into the laboratory with good quality pressure regulators and in-line microbial filters $(0.45 \mu \mathrm{m})$ where pipes enter the lab. Failure of regulators can be disastrous and they should be well- maintained and maintenance recorded for safety inspections. In many countries there are national regulations for storage and handling of pressurised gases. In particular the laboratory scientist must ensure that gas cylinders are moved safely and securely fixed (to avoid serious accidents should they be pushed over) and that regulators are regularly safety checked.

\section{Ventilation}

Air conditioners are essential to control laboratory temperatures and in extreme weather can also be a source of contamination (due to presence of water for humidification) and interference with class II cabinet airflow. Hence careful selection of the location where the air conditioner is fitted and regular servicing of the unit is important. Air conditioners with high quality filters are available and provide some control over dust and humidity in addition to temperature. Air conditioners can provide an acceptable laboratory air environment as long as personnel are meticulous about carrying out all aseptic procedures in class II cabinets and regular cleaning and disinfection or sterilization of laboratory surfaces and equipment is carried out. Filtered air (ideally HEPA filtered) providing positive pressure to clean areas, is recommended where space and resources allow. In either case a maintenance contract for regular monitoring and servicing of air-handling equipment by trained personnel from the supplier is essential to ensure that air quality is not compromised and to avoid breakdown of equipment.

\section{Electricity}

A generator for backup in case of failed power supply is essential to keep essential equipment running until cells can be put away. This is another issue to be considered when choosing the laboratory location. Emergency lighting with battery-powered lamps should be installed to avoid accidents due to sudden darkness before the generator comes on. The few seconds between power failure and generator backup may result in biosafety cabinets being briefly switched off, thus, interrupting air flow and compromising the sterile environment. It may also cause accidental spills or loss of samples.
Hence uninterrupted power supply (UPS) units should be provided for essential equipment (class II cabinets, incubators, air filtration) and to allow cell culture procedures to be completed.

\section{Specialist Areas for Handling Clean Cell Cultures} and Containing Cultured Agents

Laboratory furniture should be made of materials resistant to caustic agents and easily cleaned such as synthetic laminates or metal, preferably stainless steel. Where possible, surfaces should be impervious to water, acids, disinfectants and detergent. Wood should be avoided completely as even treated wood/board tends to warp over time, especially in extreme humidity conditions (e.g. monsoons), leaving surfaces that can harbour contaminants and are difficult to clean and disinfect. The availability of certain synthetic materials varies in different countries but granite tops for laboratory benches are inert, durable and widely available from suppliers in most countries. Avoid surfaces that are not accessible to cleaning or promote gathering of particulates. Joints between walls, floors and ceilings should be coved and sealed imperviously (e.g. silicone sealant) to enable cleaning. Windows should be avoided. If present, windows must be sealed with double layered plain glass and silicone sealant.

\section{Equipment}

Failure of equipment will impact on laboratory safety and cause loss or at least delay of important research outputs. Equipment location, installation, use and maintenance to ensure correct function should also be considered during laboratory design. Selection of the supplier is also important to address at the design stage as this can have long term effects such as:

- Delays in research work due to failure of equipment to meet local demand (e.g. inadequate autoclave capacity). - Excessive cost of consumables, maintenance and repair.

These problems can be avoided by careful specification of equipment based on local need and obtaining accurate information from suppliers on lifetime costs of equipment.

Equipment breakdown is probably the worst nightmare for many laboratories where there are difficulties with repair and replacement. In general, it is wiser to opt for simple and robust equipment design that is suitable for the task at hand, compact, easy to clean, hardy and can be serviced by the user if necessary or institutional service personnel. Equipment with sophisticated electronics is best avoided unless absolutely essential. This is especially important for laboratories that do not have a reliable supply of electricity, since fluctuations in the current and voltage can permanently damage some equipment. 
Another important factor in deciding on equivalent brands is the service provided by the local equipment dealer. Investigators will be left helpless and frustrated if service personnel are not locally accessible and cannot visit the site for long periods of time. Equipment manufacturers should be asked to provide sufficient on-site training to users for routine care of the equipment and should have more than one service engineer on call at any given time. Emergency services should be covered in a service agreement with the provider.

\section{Laboratory Operation}

\section{Laboratory Practice}

\section{General Practice in the Cell Culture Room}

This is the heart of the laboratory and requires careful attention to detail. Regardless of input air quality, all culture rooms require regular cleaning and strict monitoring of procedures. All laboratory staff using the culture room should be well-trained in laboratory maintenance. Each laboratory member should share responsibility for laboratory maintenance for which a cleaning rota could be scheduled and general laboratory activities should be supervised by a nominated member of staff responsible for laboratory fumigation, general disinfection and sterilization. In warm and humid climates, regular fumigation and pest control may be needed although frequent fumigation with formaldehyde could represent a health hazard for laboratory workers. (see Appendix 1). Pest control is important to prevent transmission of disease as discussed in Operational considerations section, and can be achieved by regular cleaning of surfaces with suitable repellent and also marking areas with insect- repellents. Any dampness or fungal growth should be treated immediately- elimination of dampness and anti fungal treatments are a good way to prevent fungus. The laboratory should be laid out with appropriate ventilation to avoid dead-spaces that can readily become damp. Care must also be taken to regulate the laboratory temperature and humidity levels (i.e. low) to inhibit fungal growth.

Given the high amount of particulates in most tropical or arid regions, all cell culture manipulations should be performed inside the class II cabinet. This also serves to protect laboratory workers from unidentified virological contamination of cell cultures [11]. For labs that do not have a "clean room", a high quality air conditioner in the cell culture room and anteroom and setting up appropriate SOPs should permit antibiotic-free long term cultures of stem cells. Dehumidifiers could be installed to reduce humidity (for example, during wet seasons) with regular monitoring of particulate counts and spore counts.
One major operational difference between research using stem cell cultures and other cells is the requirement for manual passaging by colony dissection in several protocols. This requires longer exposure of the culture to the room environment and possibly introduction of non-culture components into the culture dish. It is advisable to carry out all procedures in a laminar flow hood, use disposables and inexpensive tools (like a freshly pulled cutting capillary for each dish) as well as follow procedures outlined in Personnel section.

The main stem cell culture room should remain uncluttered and house the minimum required equipment, such as incubators, microscope, biosafety cabinets, low speed centrifuge (clinical) and laptop computers if required. Plastic covers can be used for keyboards to enable cleaning. All material brought into the laboratory should be sterilized, disinfected (typically using 70\% isopropanol in water) or put through a UV pass box as appropriate. Outer packaging is highly likely to carry contamination and should be removed in an anteroom prior to lab entry and no cardboard permitted in the cell culture clean room. Waste dishes, media and trash/waste bags should never be left overnight in the lab. All items to be discarded should leave the laboratory along with the user and should be disposed off promptly according to local rules.

As described in the section on design above, the ante-room or clean room corridor to the cell culture room acts as a first barrier to particulates and for research cell culture laboratories should be maintained in a similar way to the culture room. This can include a changing/gowning area and equipment such as fridge, freezers, storage, high end equipment, computers and desks. All reagents should be swabbed with a disinfectant such as 70\% isopropanol before they are stored in refrigerators and freezers which should also be cleaned regularly; including internal surfaces and door seals. Equipment and doors should be touched only with clean gloved hands.

\section{Personnel}

All procedures required for maintenance of aseptic technique and GCCP [3] should be adhered to strictly. Laboratories operating in tropical climates may require additional precautions built into SOPs given the high level of dust and microbial load. Footwear must be left outside the laboratory and clean footwear (e.g. overshoes) should be worn once inside the anteroom. Hands must be washed thoroughly with anti-bacterial soap or gels before donning laboratory wear. All coats, smocks, caps, masks, gloves etc. worn in the laboratory MUST BE individually packed and autoclaved before use. Procedures that are required only for cGMP manufacturing in temperate or dust-free zones may be useful to adapt even for basic research laboratories in the tropics. The local environmental conditions and research requirements 
(for example- long-term antibiotic-free culture) will determine the level of precaution to be used.

Special attention should be given to the order and manner in which laboratory wear is donned and removed and this should be described in an SOP. The suggested order for donning labwear is shoes, labcoat/smock followed by mask, cap and gloves- the order is reversed when removing laboratory wear. It is important to set aside sufficient time to train new personnel in the proper manipulation of laboratory wear so that sterility is not compromised. For example, gloves should be held firmly at the wrist and pulled on in one single motion- no other part of the outer surface of the glove should be touched with bare hands. No laboratory equipment or surface should be touched with bare hands. Personnel should also be trained to refrain from touching any exposed part of their face or skin with gloved hands. Head caps and masks are a must and should be changed at least once a day. Laboratory wear worn outside the clean area should be removed and fresh, sterilized laboratory wear should be worn before entering the stem cell culture lab. Jewellery and adornments that cannot be removed should be worn beneath laboratory coats/caps or surgical tape. Laboratory coats/suits should be full-length, of material that does not shed fibres, is easily cleaned and well-fitted to the user to provide ease of manipulation and comfort during longer periods of lab work.

\section{Equipment}

Biosafety cabinets need to be monitored regularly to ensure they achieve acceptable levels of particle count and provide operator protection (e.g. BS5726). Incubators should be regularly cleaned and disinfected or sterilized on a regular basis. Incubators on wheels with extended gas tubing or other arrangements should be considered to facilitate maintenance and regular laboratory cleaning. Equipment calibration and testing procedures should be included in laboratory SOPs and along with equipment maintenance and repair should be documented in an equipment log. Finally, all service personnel entering the laboratory should receive instruction in special laboratory hazards and any necessary procedures for working in clean areas e.g. gowning, hand disinfection.

\section{Reagents and Supplies}

A valuable rule of thumb is to recognize that suppliers will not necessarily consider the needs of local users and it is important to check everything that comes into the laboratory for evidence of its suitability for use. Well-characterized and quality controlled stocks of cells, tissues, media and reagents must be obtained only from reliable sources. 'Service level agreements' (SLA) can be used with regular suppliers to establish a range of user requirements and minimum delivery times. These can also specify a requirement to notify users of delays, shipment conditions and arrangements for topping up ice or dry ice packages, return and replacement policy, provision of certificates of analysis, material data, quality control and safety statement, payment arrangements for client, and spare parts and servicing commitments for equipment purchases. However, a number of these items may be covered in the suppliers 'standard terms and conditions' and will not need to be duplicated in an SLA.

Important properties of reagents should be checked by provision of certificates of analysis. In some regions delivery times for imported reagents, especially non-standard ones, may be several weeks. This forces the researcher to plan ahead and establish sufficient laboratory stocks of reagents where possible. Hence, it is important to check the expiry date of reagents to avoid running out of critical reagents. While many reagents can be used past the expiry date, the researcher should be alert for possible problems that this may cause. This necessitates thorough staff training, detailed record- keeping practices and monitoring the suitability of any out-of-date materials if these have to be used. The routine monitoring of quantities and expiry dates of laboratory reagents is basic good laboratory management practice. Simple tools can be used to 'flag' the need to reorder reagents; such as a marker, or "two-bin" system whereby reaching the marker or second storage "bin" prompts reorder. Numbering aliquots and using them in reverse order is another easy way to keep track of the number remaining that will alert laboratory operators to the need for a new batch of the reagent to be prepared or ordered. Such systems are particularly important where new stock may need to be imported or where pre-use quality control must be performed. Pre-use testing is especially important for materials that are highly variable, such as calf serum and some other critical biological reagents.

Often laboratories experience long delays in supply and poor supplier responses in case of problem shipments. This may necessitate an attempt to source reagents from other laboratories or suppliers. Extreme caution should be exercised in this regard. The history of shipping and storage conditions all through the life of the reagent should be traceable. Alternative sources of materials should be tested for suitability prior to abandoning stocks from the main supplier, so that the switch to the alternative is smooth and effective. Such switching of source on a regular basis should be avoided and it is important to establish suitability of an alternative supplier before an emergency situation arises.

\section{Cryostorage}

\section{Contamination Control}

The requirement for good ventilation often compromises storage areas by exposing them to high levels of contamination [17] and therefore, secondary containment and careful 
disinfection of cryovials on removal from liquid nitrogen storage is recommended. Depending on levels of environmental contamination it may also be necessary to very occasionally (e.g. every 10 years) empty, thaw out and clean the storage vessels, however, it should be recognised there are significant risks in moving all stored material out of a laboratory's main storage vessels.

\section{Staff Safety}

The primary concerns regarding safe management of liquid nitrogen storage are frostbite burns from skin contact with liquid nitrogen and asphyxiation due to exposure to low oxygen levels when nitrogen gas is released from vessels. The user can become unconscious rapidly within seconds with little warning and laboratory workers have been killed due to nitrogen asphyxia in storage areas. Staff should be trained in safe handling of liquid nitrogen and safety procedures including emergency procedures and use of protective aprons, gloves and masks. The procedures for working in the storage area should be documented. Storage areas should be fitted with oxygen alarms and staff may also have access to alarms which are triggered should they collapse.

\section{Inventories and Documentation}

Accurate documentation of stored material is vital to ensure readily available supplies of cells, avoid failure to replenish frozen stock cultures and potential loss of cell lines. A database maintained in hard copy or electronic form should be established to provide information on stored material. The database should be duplicated or otherwise backed up to ensure that loss of the primary records is not catastrophic. Inventory and storage monitoring programs and bar-code labeling systems can be used where affordable to allow accurate monitoring and easier review of reagents and supplies used as well as for tracking vials in cryostorage.

\section{Contingencies}

Emergency supplies of liquid nitrogen should be easily available in case high usage or accidental loss requires emergency replenishment. Failure of key equipment or other catastrophic accidents could lead to loss of material held at one site. Therefore careful consideration should be given to splitting stocks of key material either at different points on the same site or providing a distant storage site, such as collaborating institutions or commercial storage companies. Second site storage should be established and monitored to ensure the quality of storage is suitable and consistent.
Cell Culture Expansion, Preparation of Cell Banks and Quality Control

Advice on fundamental principles of good cell culture practice has been published [3] and there is helpful general guidance on cell culture and cell banking protocols in the literature $[8,14]$. In addition there is also specific consensus guidance on the banking of hESCs [1] that are also applicable to iPSC lines. Staff should be aware of good cell culture practice and key procedures should be captured in laboratory SOPs. New cell cultures used in the laboratory for the first time should be quarantined in a separate room or a separate cabinet and incubator used for their culture until mycoplasma testing [16] has been performed (see below).

Procedures for quality control of cell lines and banks have been published [13] and are also dealt with in the references already given [1, 14]. In setting up a new laboratory the resources, skills and equipment required to carry out routine quality control should be included. In particular these should include a means of carrying out routine mycoplasma testing due to the ability of this organism to spread between cultures rapidly and to cause permanent damaging effects to cell lines. Feeder cells used for maintenance of stem cell lines should also be batch tested for mycoplasma and sterility as a minimum. If mouse embryonic feeder cells are being derived, the microbiological status of the source animal colony should be monitored.

Stem cells or transformed cell lines are often cultured long term for research. Stem cells are highly susceptible to karyotypic changes, which is undesirable, especially as karyotypically abnormal cells may not form good developmental models and also are highly unlikely to be acceptable for regenerative medicine. Hence it is crucial to generate banks of early passage stem cells after checking their karyotype and differentiation properties. Experimental cultures can then be grown for shorter durations and replenished regularly with a fresh stock, thereby reducing the likelihood of karyotypic changes.

\section{Dealing with Contamination}

Contamination of cell cultures is one of the most common causes of lost time and wasted resources and can cause serious delays to research work. Occasional loss of cultures due to bacterial or fungal infection probably occurs in all laboratories and is not a serious cause for concern provided affected cultures are discarded immediately in a way that will prevent infection of other cultures or growth media. Routine screening for mycoplasma and microbial monitoring of the laboratory environment will help alert to serious issues so they can be dealt with quickly. It is important that individuals in the laboratory report contamination immediately. Otherwise this could lead to very serious widespread problems in the 
laboratory and could bring all work to a standstill. Where a more serious contamination event occurs or where it appears to have occurred in the culture work of a number of individuals in the laboratory then there should be an open discussion amongst laboratory staff and an investigation to try to trace the source. In such cases it is important to discard affected cultures and any opened growth media to try to exclude the most direct sources of contamination. Where infection is very widespread full laboratory closure with discard of all cultures and their media and laboratory cleaning and disinfection may be required with cautious restart of culture with regular testing for the contaminant (for a fuller description see [12, 18]).

\section{Documentation, Training and Monitoring}

\section{Documentation}

Throughout this article the importance of specific documented protocols (SOPs) has been emphasised for a range of laboratory procedures. It is helpful to organise these within a structured set of documented procedures that typically might include:

- Reception of cell culture reagents and acceptance for use

- Pre-use batch testing of foetal calf serum

- Recovery of cells in to culture from liquid nitrogen storage

- Passage of hESCs/iPSCs

- Cryopreservation of hESC and iPSC lines and storage in liquid nitrogen

- Preparation of phosphate buffered saline

- Preparation of disinfectants for laboratory use

- Cleaning and disinfection of centrifuges and incubators

- Disposal of laboratory waste

- Autoclaving cell culture waste

- Periodic laboratory cleaning

- Testing, use and maintenance of class II cabinets

- Viability testing

- Mycoplasma testing

- Sterility testing

- Operation of flow cytometer and preparation of stem cell lines for analysis

This is by no means a comprehensive list but gives an idea of a basic list of core methods that might be captured in SOPs in a typical stem cell laboratory. An example of an SOP can be found in Appendix 1. Each SOP should contain similar information and be structured as follows:

1. Title.

2. Purpose. A summary of the protocols involved and their application and constraints.
3. List of materials and reagents required and cross reference to any other relevant SOPs.

4. Step by step protocol.

5. Annotations in the form of technical advice notes.

6. Safety issues and procedures.

7. Relevant references.

There will typically be a hierarchy of documentation within the cell culture laboratory and this often includes the following:

- High level policies on aspects of laboratory work (this may include laboratory safety rules, staff training policies and programmes, and in some circumstances where formal quality standards are required, this may include a quality manual to describe the standards adopted)

- SOPs for laboratory procedures

- Record templates and log books for documenting results and processes and training carried out

The complexity of such systems will vary depending on the role of the laboratory. Clearly a laboratory processing material for use in humans or manufacture of products will require more detailed documentation to record laboratory procedures to meet the appropriate quality standards.

Similar documentation is relevant for safety procedures (e.g. handling toxic chemicals, GMOs, flammables, dealing with infectious/toxic spills) and records (e.g. records of dangerous chemicals storage, pathogen storage, staff training) and this will be prescribed in national laws/guidelines and local institute rules.

\section{Training}

Staff training provides staff with a level of competency to enable them to fulfill their duties precisely and effectively; qualities fundamental to the successful operation of a cell culture facility. These competencies can be captured in a set of training records. Staff should have a good biological sciences background on which to build a knowledge framework around the theoretical and practical processes required for cell culture in a quality assured environment

Training should include:

- Competency in laboratory safety.

- Competency in the aseptic techniques required for cell culture and related processes.

- Competency in cell banking.

- Competency in characterisation and safety testing of cells and cell lines.

- Competency in quality control and record management.

- Competency in the use of laboratory equipment.

Other related training requirements i.e. specialist training in specific technologies. Training should be a dynamic process whereby competencies are re-assessed 
at certain defined time periods to ensure that the required skill sets are not lost. The maintenance of complete up to date training records should be held by the laboratory head or the laboratory quality manager. These records should form part of the documentation for the cell culture laboratory.

\section{Monitoring}

In order to check that the systems of working are being used appropriately, audits may be performed. These are more usually carried out in research laboratories for safety aspects of laboratory work but audits may also be used where it is required to demonstrate adherence to laboratory protocols i.e. when work is performed to meet a formal quality standard e.g. ISO9000, ISO17025, ISOP13485 [for a general reference see the International Standards (http://www.iso.org/iso/home. html)]. Such audits should be performed and reported by individuals managerially independent of the work being audited. This will clearly be relevant for provision of cells for human use but in the future may also be relevant to stem cell laboratories using stem cell lines to provide toxicity data on candidate drugs (http://203.200.89.92/dst/rti-info/rti-glp.pdf)

\section{Ethics}

For the use of any human or animal tissue, certain ethical issues arise that are often regulated under national law. Researchers must not only familiarize themselves with their own national legislation and guidance but also any specific legislation that may require their attention when collaborating with researchers in other countries. Consequences of failing to meet such legislation can be serious. Furthermore, in the case of hiPSC lines from very rare diseases it may be likely that even the anonymised cell lines could be identified with particular families if the country of origin of the original tissue is known. Researchers working with stem cell lines should carefully consider their responsibility to sustain donor anonymity.

\begin{abstract}
Acknowledgements MI and AS thank the Department of Biotechnology, Government of India and the Jawaharlal Nehru Centre for Advanced Scientific Research for laboratory and research support; LH and GS acknowledge the UK Stem Cell Bank. The authors would like to acknowledge feedback received from several stem cell trainees.
\end{abstract}

Conflict of Interest Statement The authors declare no potential conflicts of interest.

Open Access This article is distributed under the terms of the Creative Commons Attribution Noncommercial License which permits any noncommercial use, distribution, and reproduction in any medium, provided the original author(s) and source are credited.

\section{Appendix 1. An Example of a Standard Operating Procedure (SOP)}

Title-Laboratory Fumigation Protocol

\section{Introduction/Purpose}

Fumigation should be performed as part of preparation of new lab areas prior to the initiation of cell culture work or as a remedial action following contamination events. Fumigation is achieved by mixing chemicals (KMnO4 and $\mathrm{HCHO}$ ) that generates toxic Formalin fumes causing the death of organisms. The use of formaldehyde is hazardous to lab workers and must be carried out with care.

\section{Requirements}

Disinfectants and 70\% alcohol for lab cleaning, Glass beakers, Plastic sheets, Blotting paper, Potassium Permanganate $\left(\mathrm{KMnO}_{4}\right), 40 \%$ Formaldehyde $(\mathrm{HCHO})$, tape (for sealing the doors to the room being fumigated), warning notices (for health and safety purposes, to inform colleagues that this procedure is taking place) and any other SOPs relating to the fumigation procedure.

\section{Planning}

1. Discuss with laboratory head, lab manager and lab users how long it will take to complete ongoing experiments and discard/freeze cultures. This may not be feasible in an emergency, such as, when the laboratory is experiencing severe contamination. Generally, expect the laboratory to be inoperable for at least 3-4 days during fumigation.

2. If any cultures must be continued, arrange to have them moved to a clean and controlled laboratory environment.

3. Plan to have equipment, biosafety cabinets, air conditioners, clean room, HEPA filters etc. cleaned and serviced before fumigation.

4. Arrange for storage of refrigerator and freezer contents to alternative sites if these are part of the area to be fumigated.

5. Move liquid nitrogen tanks outside the fumigation area.

6. Consult clean room service personnel regarding appropriate precautions to take so that filters etc. are not damaged during fumigation.

7. Remove sensitive equipment out of the fumigation area.

8. Any new material to be stocked or equipment to be installed in the laboratory should be brought in before fumigation.

\section{On the Day of Fumigation}

1. Remove cultures and switch off electrical supply to all equipment and $\mathrm{CO} 2$ supply.

2. Defrost and clean refrigerators and freezers and leave open. 
3. Drain and clean water baths, incubators and leave open.

4. Clean and swab all surfaces, drawers and cabinets.

5. Autoclave shelves and accessories that can withstand autoclaving.

6. Seal windows and openings where formaldehyde could leak into adjacent areas where people work. Notify local workers that fumigation is about to take place.

\section{Procedure}

1. Weigh and keep separately one part of $\mathrm{KMnO} 4$ and three parts of HCHO per fumigation beaker. Generally, for a 100 square feet area $15 \mathrm{~g}$ of $\mathrm{KMnO} 4$ and $45 \mathrm{ml}$ of $\mathrm{HCHO}$ is used. For a single hood, $3 \mathrm{~g}$ of $\mathrm{KMnO} 4$ along with $15 \mathrm{ml}$ of HCHO is used.

2. Place a glass beaker containing appropriate amount of $\mathrm{KMnO}_{4}$ on a plastic-lined blotting sheet to catch unwanted spillage.

3. Ensure all electrical supply is switched off. Use battery operated lights if required.

4. Keep formaldehyde tubes labeled and ready in the order in which they will be used. As soon as the formaldehyde is mixed, noxious gas starts to effervesce, which is highly injurious to health. Hence, it is advisable to start with the innermost room first and finish with the outermost room.

5. Ensure that all other personnel have left the laboratory.

6. Add formaldehyde to the respective beakers in the predetermined order. Leave the room immediately and seal all exits. Ensure that a prominent notice is displayed, to prevent anyone trying to gain entry.

7. After at least $24 \mathrm{~h}$ open the laboratory and let the fumes vent. Ensure all personnel take appropriate precautions against the fumes. Ammonia solution can be placed in beakers across the laboratory to facilitate quicker absorption of fumes.

8. A day after venting out the fumes the laboratory air conditioning and equipment can be switched on and prepared for use.

9. It is advisable to place clean test cultures in incubators for at least a day after startup to ensure that no toxic fumes remain in the laboratory that may harm cultures.

\section{Notes on Safety Measures}

Inadequate ventilation can cause irritation to the eyes. In case of emergency eyes should be irrigated with water and immediate medical treatment should be sought. Additional laboratory-specific safety measures must be incorporated into the sample SOP based on local conditions.

\section{References}

1. Andrews, ISCBI, et al. (2009). Consensus guidance for banking and supply of human embryonic stem cell lines for research purposes; the international stem cell banking initiative. Stem Cell Review and Reports, 5, 301-314.

2. Chadgee, D. D., \& Le Maitre, A. (1990). Ants: Potential mechanical vectors of hospital infections in Trinidad. Transactions of the Royal Society of Tropical Medicine and Hygiene, 84, 297.

3. Coecke, S., Balls, M., Bowe, G., Davis, J., Gstraunthaler, G., Hartung, T., et al. (2005). Guidance on good cell culture practice. A report of the second ECVAM task force on good cell culture practice. ATLA, 33, 1-27.

4. EU GMP (1998). EU Commission for European Communities. The rules governing medical products in the European Community. Good manufacturing practice for medicinal products volume IV, April 1998 with additional annexes and updates 2004.

5. FDA GMP (2004). Title 21, code of federal regulations, part 210: Current good manufacturing practice in manufacturing, processing, packaging or holding of drugs; general. US FDA, April 1st 2004.

6. Fotedar, R., Shriniwas, U. B., \& Vermer, A. (1991). Cockroaches (Blattella germanica) as carriers of microorganisms of medical importance in hospitals. Epidemiology and Infection, 107, 181187.

7. Healy, L., Hunt, C., Young, L., \& Stacey, G. N. (2005). The UK stem cell Bank: Its role as a public research resource centre providing access to well-characterised seed stocks of human stem cell lines. Advanced Drug Delivery Reviews, 57, 1981-1988.

8. Hunt, C., Timmons, P., Day, J. G., \& Stacey, G. N. (2007). In J. G. Day \& G. N. Stacey (Eds.), Cryopreservation of hEScs by vitrification. Methods in molecular biology, vol. 368: Cryopreservation and freeze-drying protocols (pp. 39-58). Totowa: Humana.

9. Rady, M. H., Abdel-Rauof, N., Labib, I., \& Merdan, A. I. (1992). Bacterial contamination of the house fly, Musca domestica, collected from Ciaro hospitals. Journal of the Egyptian Society of Parasitology, 22, 279-288.

10. Stacey, G. N. (2004). Cell line banks in biotechnology and regulatory affairs. In B. Fuller, E. E. Benson, N. Lane (Eds.), Life in the frozen state. Taylor-Francis.

11. Stacey, G. N. (2007). Quality control of human stem cell lines. In J. Masters, B. Palsson, \& J. Thomson (Eds.), Human embryonic stem cells (human cell culture-volume 6) (pp. 1-22). Tokyo and New York: Springer.

12. Stacey, G. N. (2010). Cell culture contamination. In I. A. Cree (Ed.), Cancer cell culture: Methods and protocols. Springer Science + Business media LLC, in press.

13. Stacey, G. N., \& Auerbach, J. M. (2007). Quality control of stem cell lines. In I. R. Freshney, G. N. Stacey, J. M. Auerbach (Eds.), Culture of stem cells (pp 1-22). John Wiley \& Sons.

14. Stacey, G. N., \& Masters, J. R. (2008). Cryopreservation and banking of mammalian cell lines. Nature Protocols, 3, 1981-1989.

15. Vranch, S. (2007). Facilities for animal cell culture. In Stacey \& J. Davis (Eds.), Medicines from animal cells. Chichester: Wiley.

16. Young, L., Sung, J., Stacey, G., \& Masters, J. R. (2010). Detection of mycoplasma in cell cultures. Nature Protocols, 5, 929-934.

17. Fountain, D., Ralston, M., Higgins, N., Gorlin, J. B., Uhl, L., Wheeler, C., et al. (1997). Liquid nitrogen freezers: a potential source of microbial contamination of hematopoietic stem cell components. Transfusion, 37, 585-591.

18. Stacey, G. N. (2011). Cell culture contamination. Methods in Molecular Biology, 731, 79-91.

\section{General Stem Cell Information}

http://www.stembook.org/

http://stemcells.nih.gov/ 


\section{Cell Culture}

http:/www.sigmaaldrich.com/life-science/cell-culture/learning-center/ ecacc-handbook.html

http://media.wiley.com/product_data/excerpt/59/04716293/0471629359.pdf

\section{Regulations and Guidance}

http://www.gmp-compliance.org/eca_index.html

http://www.oecd.org/document/51/0,3746, en 264934381

4408051_1_1_1_1,00.html

http://www.ich.org/

\section{Databases}

http://www.hescreg.eu/

http://www.umassmed.edu/iscr/index.aspx

\section{International Initiatives}

http://www.stem-cell-forum.net/ISCF/

http://www.hsci.harvard.edu/research/ips-core-facility

\section{Cryopreservation}

http://www.ncbi.nlm.nih.gov/pubmed/21566712

\section{Gowning}

http://www2.dupont.com/Personal Protection/en US/assets/downloads/ cleanroom/2010/DCC10 16304 Don-Doff Poster rd1.pdf http://www.cintas.com/PDF/CleanroomResources/Ask_Jan_SEP07.pdf

\section{Environmental Monitoring}

http://www.who.int/vaccines-documents/DocsPDF/www9651.pdf contains an SOP for environmental monitoring

http://www.pharmpro.com/articles/2010/06/clean-rooms-EnvironmentalMonitoring-Maintaining-a-Clean-Room/ 